\title{
How We Do It: A DIY Smart Phone based ENT Endoscopy
}

\author{
Shyam Duvvi, ${ }^{1}$ P Neelapala, ${ }^{2}$ A S Duvvi, ${ }^{3}$ B Nirmal Kumar ${ }^{4}$
}

\begin{abstract}
We present a simple method of preparing a smart phone based ENT endoscopy for OPD clinic and for on call ENT to provide emergency ENT care in Accident and Emergency department when required. In our experience, this method is efficient, economical and ensures mobility and flexibility of remote visual inspection and documentation. This aids in better care for patients especially where there is no easy access to traditional ENT treatment room facility. This provides a fast and costeffective solution to simultaneously view capture and save photos and videos with a smartphone and, if required, send these pictures to oncall Consultant. This enables quicker identification, documentation and support regardless of location.

Keywords:

Smartphone; Endoscopy; Otolaryngology
\end{abstract}

$O$ mart phone technology is becoming increasingly integrated into medical care. Recent advances and the widespread availability of smart phones have ushered in a new wave of innovations in healthcare. ${ }^{1,2,3,4 .}$ Mobile health ${ }^{5}$ is a term used for the practice of medicine and public health and is supported by mobile devices, such as mobile phones, smart phones, tablet computers, and PDAs (personal digital assistants). Mobile health has emerged as a sub segment of electronic health.

The aim was to transform or use a smart phone as video endoscopy purpose. To make this happen we need to connect the smartphone's camera to the endoscope. The technical part is simple we used a modified commercial telescopic adapter to connect the smartphone.

We describe a simple technique and present our initial experience how a smartphone can be used for

1 - Department of ENT, Tapani Hospital, Rajahmundry

Andhra Pradesh

2 - Department of Gyneacology, Tapani Hospital,

Rajahmundry Andhra Pradesh

3 - A Level Student, Tapani Hospital, Rajahmundry Andhra

Pradesh

4 - Department of ENT, Wrightington, Wigan and Leigh

NHS Trust, Wigan lane, Wigan, UK

\section{Corresponding author:}

Dr Shyam Duvvi

email: sduvvi@rediffmail.com otorhinoscopic image capturing and management, as well as its application in ENT. This method has helped in our unit where there are patients especially without easy access to traditional ENT treatment room facility in providing emergency services.

\section{Materials and Methods}

This requires a smart phone, endoscope, portable battery powered light source, a modified telescopic adaptor which is available commercially in the market to attach to smart phone for holding the endoscope (Fig. 1).

\section{Discussion}

Our aim is to introduce a convinient method to carry ENT Examination quick, simple even at the bed side without any need for sophisticated traditional equipment. This was very useful in treating patients in patients in Accident and Emergency department and dealing with cross referrals from non-ENT wards in other wards.

A survey on out-of-hours facilities in otolaryngology by Moorthy etal ${ }^{6}$ has shown that not all ENT units have appropriately equipped out-of-hours facilities and there is a need for nationally agreed guidelines stating the minimum equipment and assistance required to provide a safe and adequate service. 

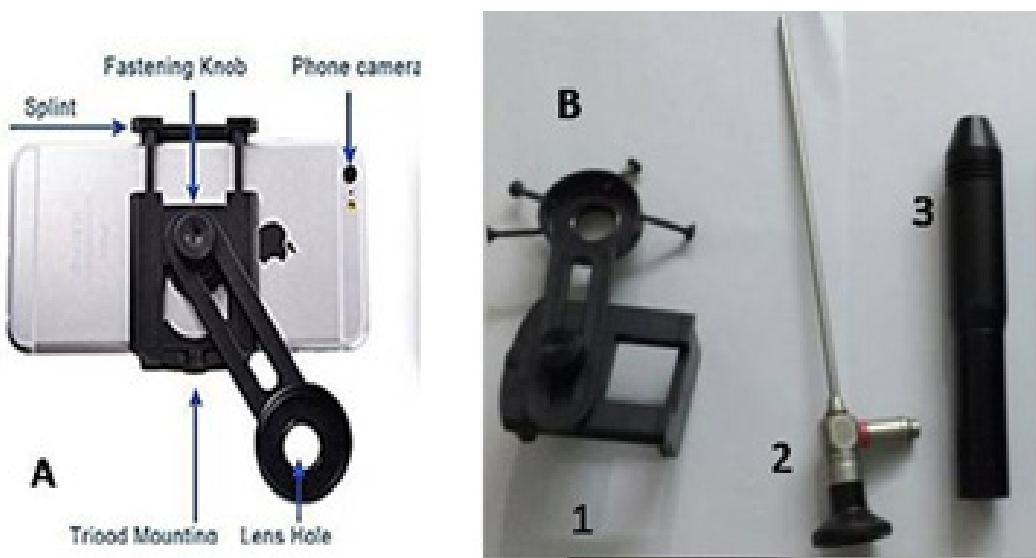

4

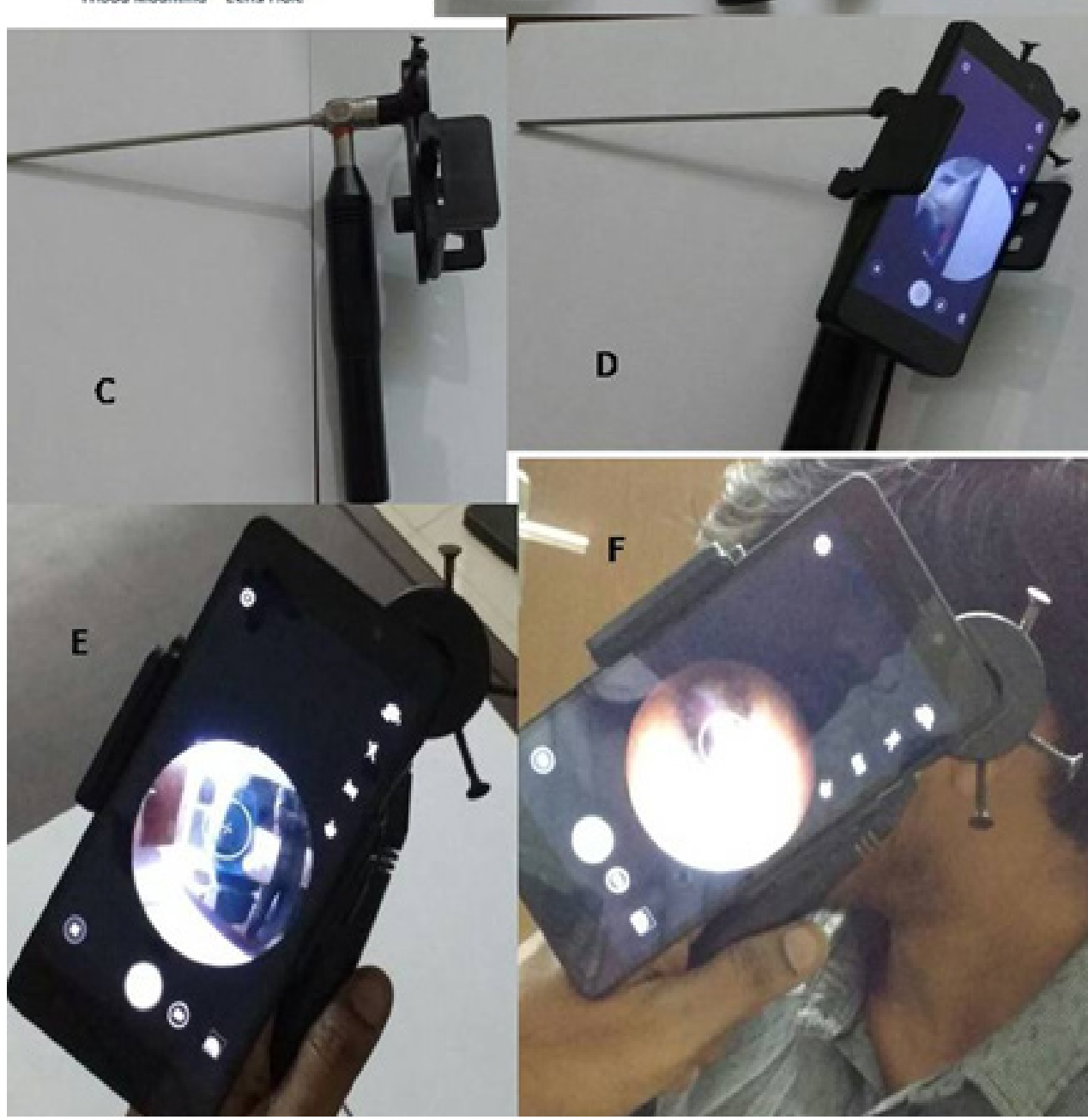

Fig. 1. ENT smart phone endoscopy unit: (A)Smart phone telescopic adaptor and its parts. (B) 1. Adaptor 2. Endocope 3. Portable light source 4. Smart phone. (C) Assembly of scope and adaptor. (D) Scope adaptor and phone as one unit. (E - F) usage of the portable Smartphone endoscope. 


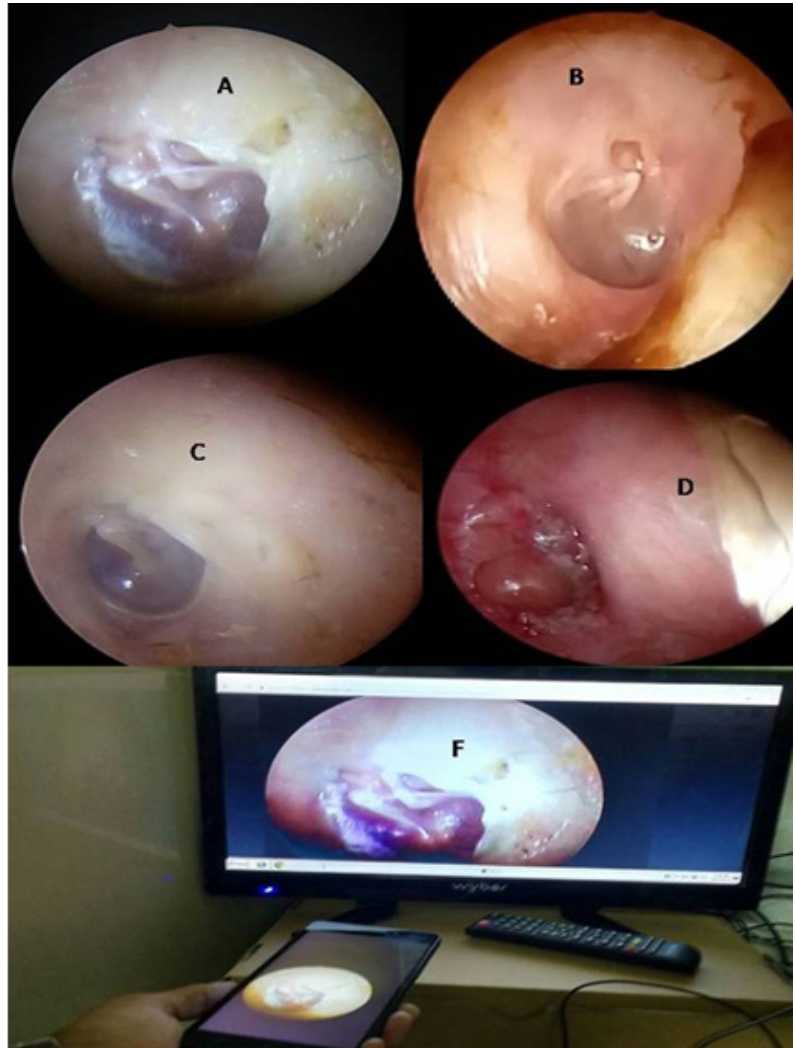

Fig. 2. Smart phone Oto-endoscopy pictures (A-D) (F) Mira cast/ chrome cast display on monitor

In conjunction with rigid scope the adaptor provides a fast and cost-effective solution with a smart phone in helping capturing saving photos and videos if required send these to a third party for quicker identification and documentation.

The DIY smart phone based endoscopy comprises an endoscope with quick attach-release coupling adaptor to fit for all smart phones. The coupler case acts as an endoscopic holder for the smart phone and establishes an optimal connection between the endoscope and the smart phone camera. (Fig. 1)

The quick-release coupling adaptor is modified from a commercially available telescopic adaptor by placing three diagonally based screws which will hold the endoscope to the smart phone camera surface in conjunction all the standard features offered by modern smart phones can be utilized. These include image and video recordings, image zoom, select focusing, image and data transfer via e-mail, Mira/chrome cast data
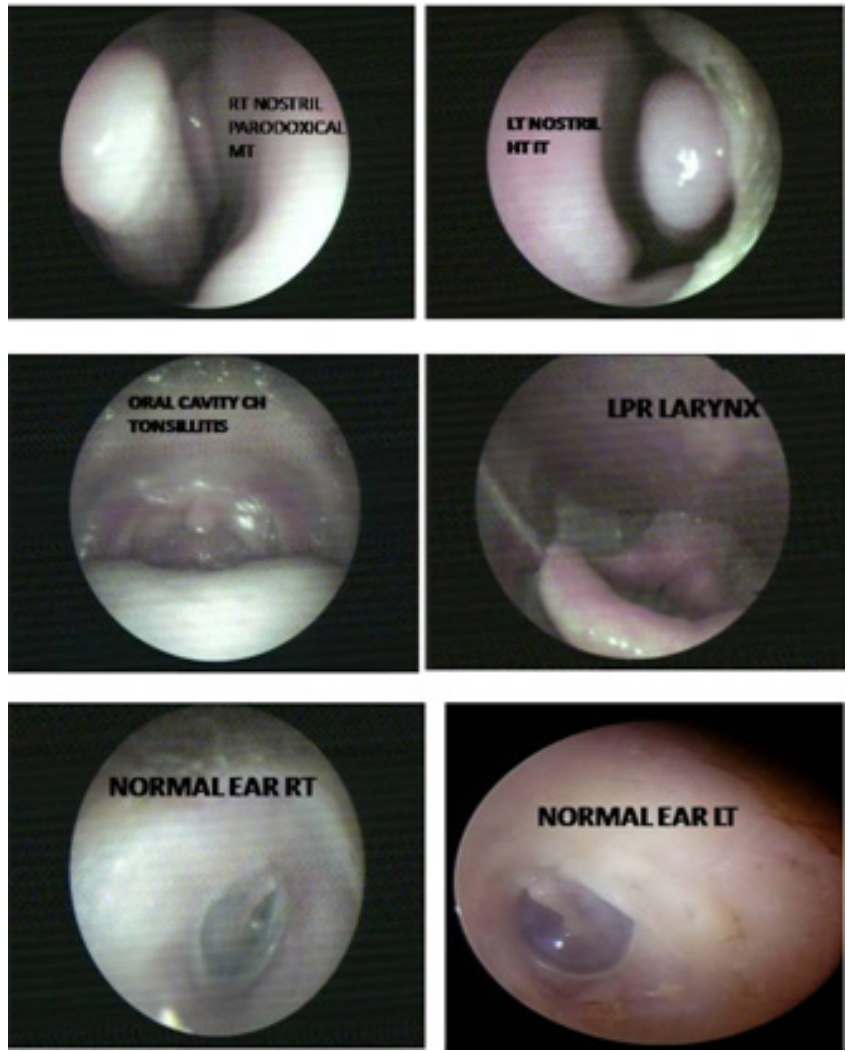

Fig. 3. Traditional endoscopy pictures using routine endoscopic camera for comparison

transfer function and much more. Moreover, various new smart phone apps (eg Pics Art, AVS Editor) are available for optimizing camera operation and image and data processing.

To maintain patient confidentiality information from the remote site about the raw data without patient personal details using a unique ID no and about the clinical condition were transmitted through the $3 \mathrm{G}$ network to a consultant for a primary diagnosis. With informed consent all the information gathered through this method will pass through Hospital Internet gateway mobile system and hard ware only

No further adjustment is necessary when the smartphone is combined with the endoscope. A modern smartphone with good image quality combined with the high-power and portable battery light source with good illumination will provide meaningful examination recordings. In our experience we use $13 \mathrm{MP}$ (megapixel) camera with $4160 \times 3120$ pixels in native mode $4: 3$ with 
focal length/2.0 aperture this gives reasonable good images.

In order to see the patients who are inaccessible to ENT treatment room we have prepared and used this DIY smart phone based ENT endoscopy for the management of common emergencies eg. Ear pain, Nose Bleed, Throat pain/quinsy. This method of examination is helpful mainly in stable patients rather than acutely ill or uncomfortable or anxious patients.

As per the protocol, after morning ward rounds the respective on call specialist trainee or foundation doctor can discuss with seniors and ensure that the steps taken for the patients seen on the on call were appropriate as this method has better visualization and documentation flexibility. The smart phone ENT Endoscopy Unit along with Mobile is a dedicated set. In order to avoid contamination can be cleaned easily and the endoscope sterilized and maintained in good condition with a daily record. A detailed search of literature and books on ENT surgery found no prior similar setup descriptions of this simple technique.

This method of DIY smart phone set up is very cost effective than the traditionally available adaptor from a standard equipment providers. ${ }^{7}$ The total cost of our unit with mobile and light source accounts to INR 16000 (USD 250 approx) the traditional standalone adaptor 7 unit itself amounts to INR 50000 (USD 780).

\section{Conclusion}

In conclusion, we present this as a simple and practical method that improves access to the basic ENT endoscopy which is now becoming a norm for examination in ensuring safe practice. This technology based instrumentation is widely available and used in Asia and Africa with cost effectiveness and helps improving patient centered approach.

\section{Acknowledgments}

To Miss Asha Sree Duvvi A level observer student for putting the kit together.

\section{References}

1. Thomale UW, Knitter T, Schaumann A, Ahmadi SA, Ziegler P, Schulz M, Miethke C. Smartphone-assisted guide for the placement of ventricular catheters.Childs Nerv Syst. 2013 Jan; 29(1):131-9

2. Sohn W, Shreim S, Yoon R, Huynh VB, Dash A, Clayman R, Lee HJJ. Endockscope: using mobile technology to create global point of serviceendoscopy. Endourol. 2013 Sep; 27(9):1154-60

3. Avidan A, Shaylor R, Levin PD. Smartphone assisted laryngoscopy: a new technique to overcome light failure in a laryngoscope. Anesth Analg. 2013 Nov; 117(5):1262-3

4. Lee M, Savage J, Dias M, Bergersen P, Winter M. Box, cable and smartphone: a simple laparoscopic trainer. Clin Teach. 2015 Dec; 12(6):384-8

5. Beratarrechea A, Lee AG, Willner JM, Jahangir E, Ciapponi A, Rubinstein A. The Impact of Mobile Health Interventions on Chronic Disease Outcomes in Developing Countries: A Systematic Review. Telemed J E Health. 2014 Jan 1; 20(1): $75-82$

6. Moorthy R, Magarey M, Joshi A, Jayaraj SM, Clarke PM. A study of out-of-hours facilities in otolaryngology: current provision and problems. J Laryngol Otol. 2005 Mar; 119(3):202-6

7. KARL STORZ SMART SCOPE.Optimal connection between endoscopes and smartphones. https://www.karlstorz.com/fj/en/ karl-storz-smart-scope.htm. 